\title{
ON SEVERAL THEOREMS OF OPERATION GROUPS.
}

BY G. A. MILLER, PH. D.

$\S 1$.

IN a recent number of the Quarterly Journal of Mathematics (vol. 28, p. 233) we proved the theorem, "Every group ( $G$ ) whose order is divisible by $p^{4}, p$ being any prime number, contains a commutative group $\left(G_{1}\right)$ of order $p^{3}$." The following proof of this theorem is much simpler and can readily be extended to apply to more general theorems.

$G$ contains a subgroup $\left(G^{\prime}\right)$ of order $p^{a}, a>3 . G^{\prime}$ contains a subgroup of order $p$ whose substitutions* are commutative to all the substitutions of $G^{\prime} . \dagger$ With respect to this subgroup $G^{\prime}$ is isomorphic to a group $\left(G_{1}^{\prime}\right)$ of order $p^{a-1}$. $\quad G_{1}^{\prime}$ contains a subgroup of order $p$ whose substitutions are commutative to all the substitutions of $G_{1}{ }^{\prime}$. With respect to this subgroup $G_{1}{ }^{\prime}$ is isomorphic to a group $\left(G_{2}{ }^{\prime}\right)$ of order $p^{a-2}$. Hence we may suppose the substitutions of $G^{\prime}$ so arranged that the first $p^{\beta}(\beta=0,1,2,3, \cdots, \alpha-1)$ constitute a self-conjugate (invariant) subgroup of $G^{\prime}$ and that each of its $p$ sets of $p^{\beta-1}$ substitutions, in order, is transformed into itself by all the substitutions of $\mathrm{G}^{\prime}$.

If we suppose $\beta=2$ each of the $p$ sets contain $p$ substitutions. The substitutions of $p-1$ of these sets must be transformed, by all the substitutions of $G^{\prime}$, according to the cyclical group of order $p$ or according to identity. Those in the first set are known to be transformed according to identity. Hence each of these $p^{2}$ substitutions must be commutative to at least $p^{a-1}$ substitutions of $G^{\prime}$ and the the first $p^{4}$ in the given arrangement must contain a commutative group of order $p^{3}$. This proves the given theorem.

In general, the first $p^{\beta-1}$ substitutions in the given arrangement are transformed by $G^{\prime}$ according to a group $(H)$ of order $p^{\theta}$. A substitution which is commutative to all the substitutions in the second set of $p^{\beta-2}$ is commutative to each of the given $p^{\beta-1}$ substitutions. Hence $H$ is simply isomorphic to a group whose degree cannot exceed $p^{\beta-2}$ and the maximum value $(M)$ of $\theta$ is given by the formula $\S$

* The operations are throughout represented by means of substitutions.

† SyLow, Mathematische Annalen, vol. 5, p. 588.

$\ddagger$ Ibid.

\& Cf. Dirichlet-Dedekind, Zahlentheorie, p. 27. 


$$
M=p^{\beta-3}+p^{\beta-4}+p^{\beta-5}+\cdots+1 .
$$

This proves the

Theorem I: The substitutions of a group of order $p^{a}$, where $a>p^{\beta-3}+p^{\beta-4}+\cdots+p+1+(\beta-1)$, can be so arranged that the first $p^{\beta-1}$ constitute a self-conjugate subgroup whose substitutions are commutative to the substitutions of a subgroup of or$\operatorname{der} p^{\beta}$.

By making $\beta=3$ in this theorem we readily obtain the former theorem, since the groups of order $p^{2}$ are commutative. Another important extension of the given theorem is based upon the fact that the given commutative group of order $p^{3}$ is a self-conjugate subgroup of $G^{\prime}$. The proof of this fact follows almost directly from the given arrangement. It is only necessary to consider the case when the first $p^{3}$ substitutions in this arrangement are not commutative. Since the second set of $p$ substitutions must then be transformed according to the transitive group of order $p$ they are commutative to only $p^{3}$ of the first $p^{4}$ substitutions. Hence there can be only one commutative group of order $p^{3}$, among the first $p^{4}$ substitutions, that contains the first $p^{2}$ substitutions. Since conjugate subgroups have the same properties this must be self-conjugate.

Sylow's proof of the theorem that every group of order $p^{\alpha}$ contains at least $p$ substitutions which are commutative to all the substitutions of the group applies equally to all its self-conjugate subgroups.* We may therefore use any self-conjugate subgroup of order $p^{\beta}$ for the first $p^{\beta}$ substitutions in the given arrangement of the substitutions of $G^{\prime}$. Hence the properties which we have observed in regard to the first $p^{\beta}$ substitutions belong to each of the self-conjugate subgroups of this order.

In particular, every self-conjugate subgroup of order $p$ is composed of substitutions which are commutative to the entire group. $t$ This is also a particular case of the theorem that a group of the order $p^{\alpha}$ cannot transform any of its substitutions of order $p$ into its $\alpha$ power unless $\alpha=1$. Since $p$ has primitive roots, the $p-1$ power of the transforming substitution would have to be commutative to the transformed substitution. From this it follows that the first power of the transforming substitution would also have to be commutative to the transformed substitution.

As we may consider the first $p^{3}$ substitutions commuta-

* Cf. Frobenius, Sitzungsberichte der Berliner Akademie, 1895, p. 983.

† Ibid, p. 982. 
tive, in the given arrangement of all the substitutions of $G^{\prime}$, we readily obtain from theorem I. the following

Corollary. Every group of order $p^{a}, \alpha>p+4$, contains $a$ commutative group of order $p^{4}$.

In particular, for $p=2$, we have that every group whose order is a power of 2 and larger than 64 contains a commutative group of order 16. In the given article we proved that there are seven groups of order 32 that do not contain such a commutative group. We shall presently construct a group of order 64 which contains no commutative group whose order exceeds eight. This will prove that we cannot assign a smaller value to $a$ in the given corollary, at least not without restricting the values of $p$.

Suppose that the following group $\left(G^{\prime}\right)$ of order 32 is made simply isomorphic to itself in such a way that the elements in the corresponding substitutions differ only with respect to subscripts.

$1 *$

ae. bf. cg. dh. im. jn. ko. lp. qu. rv. sw. tx. yr. zo. as. $\beta \theta$

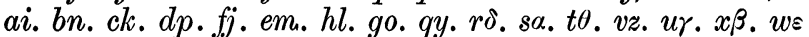

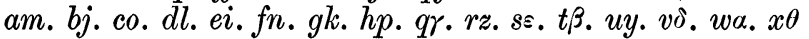
aceg. bdfh. ikmo. jlnp. qsuw. rtvx. yare. $z \beta \delta \theta$

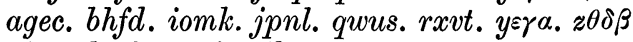

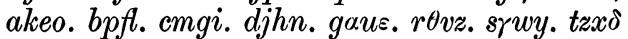

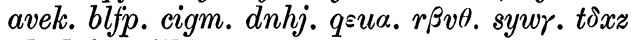

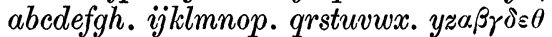
adgbehcf. ilojmpkn. qtwruxsv. y३ $\varepsilon g \gamma \theta \alpha \delta$

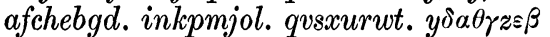
ahgfedcb. iponmlkj. qxwvutsr. y $\theta \varepsilon \delta \gamma \beta \alpha z$ ajgpencl. bohmfkdi. qzw $\theta u \delta s \beta$. rsx>vaty

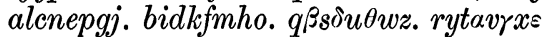

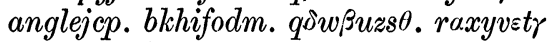

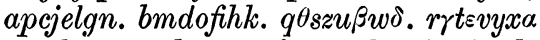
$a q . b z . c w . d \theta . e u . f \delta, g s . h \beta . i y . j r . k \varepsilon . l x, m \gamma . n v . o \alpha . p t$. $a u . b \delta . c s . d \beta . ~ e q . f z . g w . h \theta . i \gamma . j v . k \alpha . l t . m y . n r .0 \varepsilon, p x$. $a y . b v . c s . d t . e \gamma . f r . g \alpha . h x . i q . j \delta . ~ k w . l \beta . m u . n z . ~ o s . p \theta$.

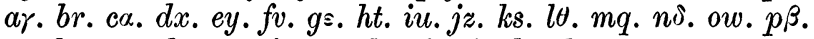

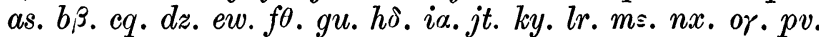

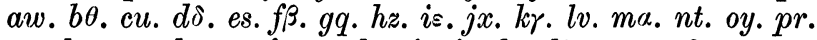
$a \alpha . b x . c y . d v . e s . f t . g \gamma . h r . ~ i s . j \theta . k q . l \delta . m w . n \beta . ~ o u . p z$. $a \varepsilon . b t . c \gamma . d r . e \alpha . f x . g y . h v . i w . j \beta . k u . l z . m s . n \theta . o q . p \delta$.

* It may be observed that these substitutions are arranged in the given manner, $i$. $e$, the first $p^{\beta}(p=2, \beta=0,1,2,3,4)$ constitute a self-conjugate subgroup of $G^{\prime}$ and the first $p^{3}$ are commutative. That each of the first $p$ sets of $p^{\beta-1}$ substitutions is commutative to $G^{\prime}$ gives additional information only when $p>2$. 


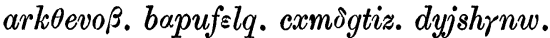

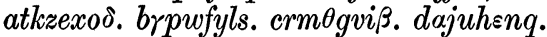
avkßerot. $b \approx p q$ falu. ctmagxio. drjwhyns.

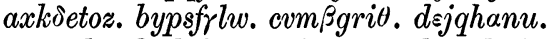

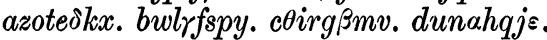

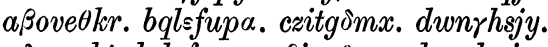

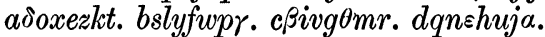
atoreßkv. bulafqps. coixgzmt. dsnyhwjr.

If we multiply the resulting group $(K)$ of order 32 and degree 64 by

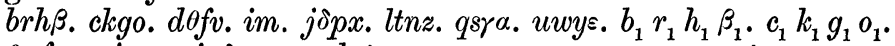
$d_{1} \theta_{1} f_{1} v_{1} \cdot i_{1} m_{1} \cdot j_{1} \delta_{1} p_{1} x_{1} \cdot l_{1} t_{1} n_{1} z_{1} \cdot q_{1} s_{1} \gamma_{1} \alpha_{1} \cdot u_{1} w_{1} y_{1} \varepsilon_{.} t$, where $t$ is of the second order and interchanges the elements of $K$ which differ only with respect to the subscript, we obtain the required group of order 64. For if this group should contain a commutative group of order $16, K$ would have to contain just 8 of its substitutions.* At least 4 of these would have to be found among the first 16 substitutions, since these constitute a self-conjugate subgroup of $K$. Hence we see that the tail to $K$ does not contain any substitution which is commutative to the substitutions of a commutative group of order 8 found in $K$. The entire group can therefore not contain a commutative group whose order exceeds 8 .

From these considerations it follows that while we can always assume the presence of a commutative group of order 8 in a group whose order is divisible by 16 , yet we cannot assume the existence of a commutative group of order 16 unless the order of the group is divisible by 128 . These facts are of practical importance in group construction since the presence of a large commutative group often simplifies the work very much.

$$
\S 2 .
$$

Theorem II : If a group contains a subgroup whose order is equal to the order of the group divided by $p$ ( $p$ being any prime number), and if this subgroup contains substitutions of order $p^{a}, \alpha>0$, all the substitutions of this order for any given value of $\alpha$, which are contained in the subgroup must generate a self-conjugate subgroup.

Let the substitutions of the given subgroup $(G)$ be

$$
1, s_{2}, s_{3}, \cdots, s_{l}
$$

If $G$ is not self-conjugate, multiply it into any substitution

\footnotetext{
* Quarterly Journal of Mathematics, vol. 28, p. 250.
} 
$(t)$ which is not commutative to it. If the transform $\left(G_{1}\right)$ of $G$ with respect to any one of these products contains a substitution ( $\left.t^{-1} s t\right)$ of order $p^{\alpha}$ which is not found in $G$ and if $s$ does not transform $G_{1}$ into itself we may form the following rectangle with $p$ conjugate rows of $l$ elements :

$$
\begin{array}{lllll}
t & s_{2} t & s_{3} t & \cdots & s_{l} t \\
t s & s_{2} t s & s_{3} t s & \cdots & s_{l} t s \\
t s^{2} & s_{2} t s^{2} & s_{3} t s^{2} & \cdots & s_{l} t s^{2} \\
t s^{p-1} & s_{2} t s^{p-1} & s_{3} t s^{p-1} & \cdots & s_{l} t s^{p-1}
\end{array}
$$

All the substitutions of a given row transform $G$ into the same group while any two substitutions from different rows transform $G$ into two different groups. As no substitutions in the given rectangle can be equal to each other the entire group would have to contain at least $l(p+1)$ substitutions. This is contrary to the hypothesis. As the opposite to the assumptions made above would in each case assume the truth of the theorem, the proof is complete.

Corollary. If $G$ is generated by substitutions of order $p^{a}$ it is self-conjugate.

In particular, if we assume that the order of $G$ is $p^{a-1}$ we obtain on important theorem due to Frobenius, which he has proved in a number of different ways as a special case of other general theorems.*

$$
\S 3 \text {. }
$$

By means of the operation $s t s^{-1} t^{-1}$ we may readily determine the smallest self-conjugate subgroup with respect to which a group is isomorphic to a commutative group, or, in other words, the order of the largest commutative group to which a given group can be made isomorphic. $\dagger$ Since $s$ and $s^{-1}$ have the same sign and a group can be isomorphic to only one commutative group of the maximum order we have the following :

Theorem III: If we make a group isomorphic to the largest possible commutative group the substitutions which correspond to identity in the commutative group are positive.

The application of the symbol $s t s^{-1} t^{-1}$ to operation groups has a two-fold importance as it simplifies the study of a group with respect to its solvability and tends to bring into prominence some of the similarities which exist between Lie's transformation groups and the older operation groups

* Cf. Sitzungsberichte der Berliner Akademie, 1895, p. 962.

+ Quarterly Journal of Mathematics, vol. 28, pp. 266-268. 
which have been developed mainly under the form of substitution groups.

It may be well to add that the symbol $s t s^{-1} t^{-1}$ has been used in substitution groups for a long time, but its use has been very limited. As far as we know its practical application to determine important properties of a group was first explained in the recent article in the Quarterly Journal to which we referred above.

GöTTINGEN,

September, 1896.

\section{NUMERICALLY REGULAR RETICULATIONS UPON SURFACES OF DEFICIENCY HIGHER THAN 1.}

BY PROFESSOR HENRY S. WHITE.

By the term reticulation I shall designate for present purposes any system of lines lying upon a closed surface, together with all the points in which these lines intersect one another. Further I shall assume that they divide the surface into portions, of which each by itself is simply connected, $i$. e., has deficiency zero. These portions of the closed surface may be termed faces, and their intersection points vertices, while each boundary line terminated by two consecutive vertices is an edge. If $F, V$ and $E$ denote the numbers of faces, vertices and edges, respectively, in a reticulation, and $p$ the deficiency of the supporting surface, then Euler's relation for convex polyedra, generalized, will be

$$
E=V+F+2 p-2 \text {. }
$$

A reticulation is clearly entitled to be called numerically regular when it has:

1. In every vertex a constant number of termini of edges; call this number $\rho+2=r$.

2. In every circuit bounding a face a constant number of edges, call this number $\sigma+2=s$.

We may for the present regard these two numbers $\rho$ and $\sigma$ alone as characteristics of a regular reticulation; there will remain for subsequent inquiry the determination of the number of essentially different types having any given set of characteristics $\rho, \sigma$, and $p$. From these three the values of $F, V$, and $E$ can be computed, as will be seen below. Counting then as one class all regular reticulations characterized by the same values of $\rho$ and $\sigma$, it can be shown that on a surface of given deficiency $p$, there can exist only a finite number of classes of numerically regular reticulations. 